\title{
Working Group Guidelines on the nursing roles in caring for patients with Crohn's disease and ulcerative colitis in Poland
}

\author{
Magdalena Golik ${ }^{1}$, Marzena Kurek², Aneta Poteralska³, Ewa Bieniek ${ }^{4}$ Anna Marynka5 ${ }^{5}$ Grażyna Pabich \\ Ariel Liebert ${ }^{1,7}$, Maria Kłopocka ${ }^{1,7}$, Grażyna Rydzewska ${ }^{2,8}$ \\ ${ }^{1}$ Centre of Endoscopy, Jan Biziel's University Hospital No 2 with Clinic for Intestinal Diseases, Bydgoszcz, Poland \\ ${ }^{2}$ Clinic of Internal Diseases and Gastroenterology, Central Clinical Hospital of the Ministry of Interior, Warsaw, Poland \\ ${ }^{3}$ University Clinical Hospital, Lodz, Poland \\ ${ }^{4}$ Gastroenterology Department, Provincial Specialist Hospital, Lodz, Poland \\ ${ }^{5}$ Gastroenterology Clinic, Autonomous Public Clinical Hospital, Szczecin, Poland \\ ${ }^{6}$ Endoscopy Laboratory, Provincial Hospital, Bielsko-Biala, Poland \\ ${ }^{7}$ Department for Gastroenterological Nursing, Collegium Medicum in Bydgoszcz, Nicolaus Copernicus University in Torun, \\ Poland \\ ${ }^{8}$ Faculty of Sciences, Jan Kochanowski University, Kielce, Poland
}

Prz Gastroenterol 2014; 9 (4): 179-193

DOI: $10.5114 /$ pg.2014.45098

Key words: inflammatory bowel disease (IBD), nursing roles in IBD, Crohn's disease, ulcerative colitis.

Address for correspondence: Magdalena Golik MSc, Polish representative in NECCO, Centre of Endoscopy with Clinic for Intestinal Diseases, Jan Biziel's University Hospital No 2, 75 Ujejskiego St, 85-168 Bydgoszcz, Poland, phone: +48 523655105 , fax: +48 523655107 , e-mail: magdago@o2.pl

\begin{abstract}
Inflammatory bowel disease (IBD), including Crohn's disease and ulcerative colitis, present a major challenge for present-day gastroenterology due to their increasing incidence, chronic nature, risk of permanent worsening of quality of life of patients, and the costs of conservative and invasive treatment. Basic and advanced nursing care are important parts of the multidisciplinary care for patients. The developed guidelines on the nursing care, which are compliant with the European guidelines published by Nurses-European Crohn's \& Colitis Organisation (N-ECCO), were adjusted to the current situation in Poland. Significant issues that are important for nursing teams have been identified, with particular emphasis on the specificity of working in centres specialising in the care of IBD patients. The Working Group paid attention to the conditions that should be satisfied in order to optimise the nursing care for IBD patients, and the necessity to develop professional and scientific cooperation with European centres within European Crohn's \& Colitis Organisation (ECCO) and N-ECCO.
\end{abstract}

\section{Introduction}

For many years, in Polish hospitals and ambulatory care units, specialists have worked based on standards and procedures developed on the grounds of reliable research and international guidelines adjusted to the Polish situation. The implementation of harmonised procedures not only facilitates the work of medical staff, but more importantly, it leads directly to the improvement of patient care. In Europe, standardised medical and nursing care also involves patients with inflammatory bowel disease (IBD) [1-7]. In Poland, on the initiative and under the auspices of the contemporary National
Consultant for Gastroenterology and the Polish Society of Gastroenterology, some recommendations were developed and published in order to update and spread knowledge on the modern principles of diagnosis, treatment, and monitoring of IBD patients [8, 9]. Inflammatory bowel disease is a chronic disease occurring in patients for most of their life, often leading to significant worsening of various aspects of quality of life and even to permanent disability [10,11]. Providing such patients with multidisciplinary care, including nursing care, is one of the fundamental conditions in the optimisation of treatment $[1,12,13]$. Close cooperation of physicians 
and nurses, and the full use of the skills of nursing staff within the "partnership" model of medical care ensures the most effective use of all possible measures that can improve the quality of care for chronically ill individuals. Until recently, the term "nursing" was used in relation to different kinds of professional tasks and operations associated with nursing activities. Over time, along with the development of various fields of medical, social, and educational science, this activity has been expanded to include aspects associated with the education, the professional development, and the whole system of organisation of nursing activities [1, 14-16].

For the affected individuals, chronic diseases, including IBD, mean that self-realisation of their needs is sometimes impossible, often requiring the assistance of other people. Care is one of the oldest and the most developed relationships. Providing support to an individual who is in a difficult situation is a part of the profession of every male and female nurse [17]. Improving the organisational structure linking the competence network, dependencies, but also ties and contacts, facilitates the provision of effective and comprehensive support to people in difficult circumstances.

Nurses-European Crohn's \& Colitis Organisation (N-ECCO) has been an active member of European Crohn's \& Colitis Organisation (ECCO) since 2007. Providing and coordinating nurse training and creating an international "network" of nurses specialising in the care of IBD patients is one of the most important goals of $\mathrm{N}$-ECCO. In accordance with their knowledge, N-ECCO assesses that nurses across Europe perform varying roles in caring for IBD patients, depending on the country-specific organisation of medical care and the situation regarding tasks, qualifications, competence, training system, and level of salary [1]. Nurses form Polish centres providing comprehensive care for IBD patients have actively engaged to work in NECCO and have started to cooperate in order to define national standards of conduct, and to create in the future an organisation of Polish nurses specialising in the care of IBD patients. Understanding the importance of the problem, the National Consultant for Gastroenterology and the Working Group appointed by the Consultant took the patronage over these activities.

\section{Aim}

The following guidelines are to identify the tasks and methods of action of all Polish nurses working with IBD patients. Inflammatory bowel disease patients have the right to expect that they will be given care that is based on the thorough knowledge and experience of staff, and that procedures will be performed in accordance with established standards.

\section{Methodology of development of the guidelines'}

On 4 December 2013, the first meeting of the Nurse Working Group - the Gastro Nurse Advisory Board took place in Bydgoszcz. Nurses experienced in the care of IBD patients from several centres in Poland were invited to attend the project. Professor Grażyna Rydzewska - the then National Consultant for Gastroenterology and Doctor Maria Kłopocka, M.D., Ph.D., a member of the Working Group of the National Consultant, took the substantive supervision of the project. At the meeting of the Working Group, important issues concerning IBD and the specificity of nursing care in this group of patients were discussed. Particular issues requiring a thorough preparation were divided among the members of the Working Group with respect to their knowledge, experience, and specificity of workplace.

At the end of January 2014, each of the members submitted their part of the work prepared based on literature data for final verification. It was compiled, and the draft guidelines were approved by all members of the Working Group. Implicit voting on particular items within the guidelines was abandoned due to the small number of persons involved in the development of the project. The entire project was submitted for substantive verification by Maria Kłopocka, M.D., Ph.D. and then presented to the then National Consultant for Gastroenterology and accepted for publication.

Nursing care should be based on providing support that consists of the following elements:

1. Mental, emotional support, which should occur in the form of empathy, showing care, and giving a sense of security.

2. Information (cognitive) support - presenting information on the situation and the position of a supported individual in an understandable and accessible way.

3. Instrumental support - providing knowledge on the procedures and promoting effective countermeasures $[16,17]$.

The guidelines were prepared with such a division. For each support category, particular arrangements were assigned. The project was divided into two parts. The first part (fundamental nursing care) provides guidelines on the care of IBD patients for nursing staff working in any setting, on the assumption that patients often benefit from the primary health care and various specialised clinics and hospital wards. Therefore, knowledge of this group of diseases and their complications is necessary in the daily nursing work. The second part (advanced nursing) presents guidelines intended mainly for staff in centres that specialise in complex care of IBD patients. Severely affected patients are directed to this type of centres. Modern, often experimental treatment 
is conducted in these centres as well. The nursing staff must, therefore, have knowledge and skills that ensure optimal patient care.

In the final part of this work some comments on the future of specialised IBD nursing have been included.

\section{Fundamental nursing care}

\section{Mental support}

\section{Outline of basic knowledge on inflammatory bowel disease}

1.1. In nursing care for IBD patients, basic knowledge on this group of diseases and awareness of the importance of establishing timely therapeutic interventions is necessary. Awareness of the key diagnostic strategies and of the main medical and surgical options available in the treatment of IBD is recommended. In any setting, nursing staff should have knowledge of these issues.

Inflammatory bowel disease includes two fundamental forms of the disease: Crohn's disease (CD) and ulcerative colitis (UC). These are chronic diseases, characterised by periods of exacerbation and remission, which most often appear in young adults and even children, and usually last for the entire life of a patient. The aetiology of these diseases in unknown, and the pathogenesis has not been fully explained. Currently, the prevailing opinion is that the diseases develop in patients with a genetic predisposition due to an impaired immune response to environmental factors, among which intestinal bacteria are the most important $[2,5,8,9]$.

Crohn's disease is characterised by transmural, intermittent inflammation, which can be located in different parts of the gastrointestinal tract, from the mouth to the anus and the perianal area. The ileo-cecum part and the large intestine are most frequently affected by inflammatory lesions. The most common lesions found at endoscopy include deep ulcers occurring along and transversely across the axis of the intestine, surrounded by unaltered mucous membrane, which gives a characteristic cobblestone-like appearance. Symptoms of the disease depend on its location, extent, and nature (phenotype). The disease may occur in the inflammatory form, intestinal stenosis form, and penetrating form (with formation of abscesses and fistulas). Initial symptoms may not be typical, which means that the disease is sometimes diagnosed after several years of delay. Patients complain about abdominal pain, diarrhoea, weight loss, and sometimes fever and chronic fatigue. With disease progression, intestinal stenosis and symptoms of obstruction, such as the aggravation of abdom- inal pain, nausea, and vomiting, may appear. Fistulas occur mostly in the perianal area, but they may also form between the bowel and urinary bladder or vagina, as well as between intestinal loops [2, 8]. Despite the use of optimal conservative treatment, approximately $70 \%$ patients require surgery at least once in their life $[3,6,8,9]$.

In the case of UC, the inflammatory process concerns the mucous membrane of the large intestine, is of continuous nature, and affects the rectum and the colon. The range of lesions may differe, up to the entire large intestine (pancolitis). Diarrhoea with the addition of fresh blood, sometimes also with mucus and pus, and with concomitant faecal urgency [5, 9], is a typical symptom of UC.

Irrespective of the form of IBD, dehydration, quantitative and qualitative malnutrition, and symptoms resulting from acute and chronic anaemia may appear in severely affected patients. In some patients, extra-intestinal manifestations affecting the joints, skin, eyes, and liver coexist or even prevail. They aggravate with increasing activity of the disease or they occur independently of it $[2,5,8,9,18]$.

Thorough medical history, physical examination, and, primarily, the results of additional tests, among which the endoscopic evaluation (colonoscopy) with biopsy and microscopic examination are the most important, are the bases for IBD diagnosis. There are also some laboratory tests - haematological and biochemical - that may confirm the occurrence of inflammation, anaemia, or nutritional disorders. Stool examination makes it possible to exclude an infection, which could be the cause of symptoms or a factor exacerbating the disease. Recently, the measurement of faecal calprotectin has been gaining great importance as a new marker of the intensity of the inflammatory process in the bowel $[2,5,8,9,19]$. Apart from evaluation of the location and the extent of lesions (especially with CD), diagnostic imaging, such as abdominal ultrasonography, computed tomography, and magnetic resonance imaging, also enable the recognition of IBD complications. Appropriate preparation of patients for endoscopic and imaging examination is one of the important tasks of nursing staff.

The main purpose of IBD therapy is to obtain and maintain full and permanent remission of the disease. The selection of pharmacological treatment, often using several drugs in various forms of administration (oral, topical, subcutaneous, or parenteral), depends on the location of inflammatory lesions, their intensity, disease duration, and also on treatment tolerance by a patient and the occurrence of possible adverse events. Formulations of 5-aminosalicylic acid, glucocorticoids, and im- 
munosuppressants are the basic groups of medicines. The progress in the treatment of IBD, which has been made in the last few years, is associated with the introduction of biological drugs to the therapy $[3,6,8$, 9]. Currently in Poland, compounds that are antibodies against pro-inflammatory cytokine - tumour necrosis factor (TNF- $\alpha$ ) are approved and used mainly within the drug programmes of the National Health Fund (NFZ). Treatment with these types of formulations is done in specialised centres meeting the criteria on staff qualifications and appropriate therapeutic-diagnostic equipment. The optimal treatment of IBD patients should also consider treatment of nutritional deficiencies, anaemia, and other complications of the disease $[3,6$, $8,9]$. In the case of failure of complete medical treatment, the desirability of surgery should be considered. In the case of UC, proctocolectomy with formation of an intestinal reservoir from a lower part of the small intestine (pouch) and reconstruction of continuity of the Gl tract is usually the surgery of choice. Procedures in the case of $C D$ are more varied. They may involve partial intestinal resection, but they also concern treatment of fistulas, drainage of abscesses, and widening of fibrous stenosis of the bowel $[3,8]$.

The effects of nursing care on the quality of life of inflammatory bowel disease patients

1.2. Nurses must be able to identify patients' needs, find coping resources that are available for them, and plan care in such a way that patients' comfort of life is maximally improved.

Contemporary nursing brings an increase of independence and competence at work. Increasing attention is paid to care based on humanism and holism.

Nursing is a unique profession. Humans during a difficult, uncomfortable period of life, i.e. during a disease, are the subjects here. Contact with ill persons requires many skills and appropriate predispositions. Due to its chronic and burdensome nature and the possibility of occurrence of different complications, IBD causes significant decrease of quality of life of patients. While performing professional duties, nursing staff have the most frequent contact with patients, and with an attitude of openness and empathy they can create the right atmosphere for establishing good contact with the patients. The nurses' ability to communicate with patients constitutes the essence of medical care, without which it is impossible to improve the quality of life of patients. Nurses should help patients in coping with helplessness, stimulate their activity and cooperation, and prepare them to make efforts to improve the state of their health. Medical staff should find time to talk and to answer the questions and concerns of patients. It is also essential to communicate with patients' families, inform them about the process of treatment, and demonstrate their important role in the overall patient care.

The assumption of the physician-nurse partnership model is to optimise patient care. Nurses have a special place and perform tasks here because, apart from their professional duties, they are a link between the doctor and the patient. This model assumes cooperation in three dimensions: nursing, therapeutic, and educational It contributes to improving the quality of life of patients and has a positive impact on the process of treatment $[1,12,17,20]$.

Quality of life is a subjective value, and only patients can describe it properly. In the case of IBD, quality of life is significantly reduced, especially during periods of exacerbation [10,11]. Estimation of the dynamics of these changes requires an analysis of the fundamental range of human activity, which can include physical fitness, emotional reaction to the disease, and social functioning. After collecting accurate information and comparing it with patients' expectations, some actions to improve the quality of life of patients may be taken. It should be remembered that the particular components rated on a scale of value and quality of life are often subjective and may be more or less important for a given patient.

The key to adequate nursing care is to identify the needs of the patients, the coping resources, and the social support available for them. The nature of the interventions taken must be individual for each patient and the situation in which they find themselves. At the stage of recognition of the condition and needs of an ill person, observations of patients, family members, own observations, medical documentation, and daily measurements of vital signs are the sources of data for nurses.

In accordance with the idea of holism, nurses taking care of IBD patients must be prepared to help solve their problems of biological, psychological, social, cultural, and spiritual nature [17]. Competent and effective action in this regard contributes to improving the quality of life. Educating patients and their families about the nature of the disease, diagnostic-therapeutic procedures, and possible complications is an important aspect of the treatment process, especially during the period after diagnosis. Inflammatory bowel disease is a chronic and - at the present stage of knowledge incurable condition. These features have a negative impact on the psyche of patients, and make their sense of independence questionable. From the time of diagnosis 
to the relative acceptance of the disease, patients often go through periods of rebellion, denial, and depressive reactions. Inflammatory bowel disease affects mostly young people, during the period of planning and execution of their own goals concerning their professional and personal life. The sooner patients accept the fact of suffering from a chronic and incurable disease, the easier it will be for them to accept the situation and continue with their life plans. In turn, a lack of acceptance may result in denial of the disease occurrence, and thus cessation of the treatment. Nowadays, also in Poland, associations of patients, their families, and supporters, as well as medical staff dealing with the issues of IBD, act more and more dynamically. Contact with other patients, the exchange of views, and support in various situations can provide a new quality in the therapeutic process. Providing information in this regard and encouraging patients to participate in meetings and to read professional magazines and websites is an important role of nurses.

An educated person accepts the proposed diagnostic and therapeutic procedures much more easily. It leads to understanding and, eventually, effective coping during different periods of the disease. Knowledge on the goals and types of specialised diagnostic tests performed in this group of diseases is an inherent part of nursing. Endoscopy is often a difficult experience for patients, due to the fear of the examination itself and its course, pain, invasiveness, and confirmation of the disease and its severity. Nurses play a key role in the mental and physical preparation of patients for a scheduled examination. When talking to patients, nurses should be able to explain the benefits resulting from the procedures, considering the selection of an optimal therapeutic option. Information on the preparation for the examination and on its course should be delivered in an objective, accurate and, most importantly, understandable way to patients. It is also important to provide a sense of safety, respect for dignity, privacy, and motivation for patients to cooperate in the diagnostic process. All these factors significantly affect the quality of the examination and its acceptance by patients [1, 21].

Health education indicating the need for lifestyle modification is another important task that depends mainly on the nursing staff. It includes not only dietary recommendations but also promotion of physical activity and elimination of risk factors exacerbating the disease. These include smoking, use of certain drugs and dietary supplements (also available without prescription, mainly non-steroidal anti-inflammatory drugs) as well as viral and bacterial infections, especially those of intestinal location (cytomegalovirus, Clostridium difficile) [1]. The impact of stress on the course of the disease is a controversial issue. Research results are inconclusive, while patients and their families are convinced that stress is an important modelling factor of the disease [22].

Recently, there have been varied opinions on the legitimacy and safety of protective vaccination. Inflammatory bowel disease patients who are often given the immunosuppressive therapy are particularly exposed to the presence of other diseases and opportunistic infections, sometimes of a severe nature. In accordance with the current standards, patients should receive the complete cycle of vaccination required before the administration of immunosuppressive drugs [8, 9, 23]. The role of nurses is to educate patients and encourage them to get vaccinated, also against flu and pneumococci.

Analysis of the available literature data, as well as own observations and professional experience of the Working Group, indicate the need for education and making significant lifestyle changes in IBD patients at the moment of diagnosis and during the many years of its course. Therefore, the efforts to popularise the problems of IBD patients in society and to improve the financing of health education as well as diagnostic-therapeutic procedures in this group of patients should be intensified. A global view of the patient and the desire to improve the quality of their life force the nursing staff to change the working model and extend the scope of professional tasks to include these new challenges. This requires an increase in the level of general and specialised knowledge, and the ability to implement new theories into the nursing practice.

\section{Communication with patients and their families}

1.3. Through direct contact with patients, the nursing staff are able to pre-medically assess patients and monitor their state of health. Competent communication with patients' families allows the nursing staff to engage them in the process of care, which positively affects the quality of life of patients.

Communication is a process necessary for the proper functioning of society. Full communication between staff of different specialities, and communication with patients, are of the utmost importance for medical care. In each of these processes, the nursing staff play a principal role.

In order to talk about communication, a basic condition must be met, i.e. at least two people must meet. These people are referred to as the sender and the recipient. In the process of communication, these roles are interchangeable. In fact, learning about a person consists of gaining information by listening and 
watching (listening - tone of voice, modulation, accentuation; watching - gestures, body movements, facial expressions, physical appearance). At the first meeting, everything is unfamiliar and new. Only a closer insight gives us a more complete picture and allows the building of appropriate relations. In relations with patients, one must not be limited to the position of the receiver, and the acquisition of information cannot be reduced to just asking questions. The initial period of disease and the exacerbation of its symptoms are the situations during which information is most frequently distorted. Communication must, therefore, be a lengthy process $[24,25]$.

Talking with patients cannot take place in hurry or in a noisy environment, and it should not be accompanied by excessive emotion. Nurses must talk in such a way as to naturally receive feedback. Building a partnership model of communication with patients allows nurses to determine an optimal nursing process, with particular emphasis on the individual needs of patients during the period of disease. The communication process must also include people that are close to patients, especially the family. Care focused on improving the quality of life of patients may be ineffective in the face of indifference of family members and loneliness of patients. Competent communication with patients' families, as well as an accessible transfer of information, allows us to include families in an effective treatment and nursing process.

Regardless of the scope of responsibilities, the nursing staff act as a link between physicians and patients. An exchange of observations on patients' condition, their behaviour, and reported problems is the key to maximum use of all relevant information, which is reflected in therapeutic decisions [26].

Improving the principles of proper communication for the benefit of staff and patients is becoming a major challenge for health care, also in Poland. Due to their occupational role and social position, the nursing staff should play a crucial role in this process.

\section{Nursing counselling}

1.4. A well-organised nursing counselling for IBD patients provides quick access to proper information and may improve the efficiency of monitoring the health of patients and the adherence to therapeutic recommendations, at the same time reducing the costs of medical care.

Care for IBD patients is a long process, often requiring modifications of the method of treatment. Contact with nurses is the fastest way to obtain proper information. An efficient nursing counselling, especially carried out by people experienced in working with IBD patients, gives patients a sense of safety and provides both the monitoring of treatment and a rapid response in the event of exacerbation or adverse effects $[27,28]$. The way of such contact is currently set at the clinic to which a patient belongs. Counselling may be carried out on a special duty, to which patients can report at any time. Contact by phone is the simplest and the best available method of contact [29]. In the majority of Polish centres for the treatment of IBD patients, this type of contact is practised. However, it seems necessary to establish some Polish nationwide standards, which requires some funding and a change of the principles of admission of patients to specialist clinics (emergency admissions omitting a queuing system). The use of modern technology in the form of telemedicine (on-line counselling, website) would enable us to provide advice not only to patients but also to employees of other units of medical care, in which the staff do not have sufficient experience in working with IBD patients. Introduction of such a method of assistance would bring tangible economic benefits resulting from the optimisation of treatment and the reduced number of unjustified outpatient visits.

\section{Creating and promoting support groups}

1.5. Nurses caring for IBD patients should be able to point out the benefits of participation in support groups and facilitate patient contact with such a group. It is advisable for the nursing staff to be actively engaged in the creation and activity of support groups.

Symptoms associated with the disease often necessitate changing the current lifestyle. Patients feel alienated, pushed to the margins of society. They are often forced to stop working, or attending school or college. In view of the complaints and limitations resulting from the disease, there may be low self-esteem and a sense of hopelessness [10, 11, 30]. The medical staff and other patients become the patient's immediate environment out of necessity. However, it appears that at the same time this may be one of the most effective forms of help. Patients who have developed the same disease understand each other best, and thus they are able to help others [22]. Nowadays, also in Poland, associations of patients, their families, and supporters, as well as medical staff dealing with the issues of IBD, are acting more and more dynamically (Polish Association Supporting People with Inflammatory Bowel Disease). Contact with other patients, the exchange of views, and support in various situations can provide a new quality 
in the therapeutic process. Providing information in this regard and encouraging patients to participate in meetings and to read professional magazines and websites is an important role of nurses.

\section{The transition of patients from a paediatric department}

1.6. Nurses caring for IBD patients should, if possible, participate in the process of growing of paediatric patients and help to smoothly prepare their transition to an adult department.

The process of transition of a juvenile patient to care where they will have to take part in the process of treatment alone is extremely difficult, and it should proceed in a long-term manner. The transfer cannot take place suddenly, overnight. A young man or woman should be prepared by professionals, mainly in the fields of nursing and psychology. Previous decisions on diagnostic-therapeutic procedures were based on the consent of parents or caregivers. With adulthood, this obligation falls on the shoulders of young, often confused men or women. The period of changing the medical team often coincides with a period of youthful rebellion, sometimes also with difficulties in education. All this may cause a sense of anxiety and a depressive reaction in young people. It makes proper treatment much more difficult. The rule of the presence of parents at the treatment process should be continued at least in the first weeks and months of independence. In order to ensure an optimal transfer of patients, close cooperation between the paediatric team and the adult care team should be discussed. The exchange of documentation, experiences, and observations between nursing teams would be a perfect solution. Development of homogeneous research tools and procedures would give a possibility to provide full information about "transferred" patients. Understanding the patients' situation at the beginning of treatment would make the planning of an optimal therapeutic process and monitoring of the disease in adulthood easier [31, 32].

\section{Information support}

\section{Communication of information about the disease}

\subsection{Communication of information about the disease} (at the level of nursing care) must be adapted to the age, emotional development, and social status of patients.

Communication with IBD patients is a fundamental aspect of nursing care, in which knowledge of the dis- ease and verbal skills in the transfer of this knowledge are crucial for patients and the diagnostic-therapeutic process. The disease usually affects young people who ask a lot of questions related to everyday life and the impact of the disease on their careers, education, social life, and intimate contacts. Nurses should tell patients not to exaggerate the impact of the disease on their lives, and, on the other hand, to be aware of certain limitations which may arise during periods of exacerbation or during complications. It is an important part of education to convince patients that complying strictly with recommendations of physicians and nurses is the basis of therapeutic success, and it allows patients to achieve and maintain remission. The ability to provide full information on nursing procedures is an integral part of care. In the case of doubts or lack of sufficient knowledge and experience, staff who are in contact with IBD patients should indicate centres where they can obtain complete professional information. Nurses should also pay attention to the patients' age, emotional state, and social status, since these may affect the reception of information provided; moreover, certain individual features of patients may be important for the planning of therapeutic procedures [24, 25, 27, 33].

\section{Diet and nutrition}

2.2. Nursing staff should have basic knowledge on dietary recommendations and possible problems with nutrition in IBD patients. Patients and their caregivers may expect from the nursing staff continuous assistance and education regarding nutrition at each stage of the disease, taking into consideration the patients' age, the form of the disease, and the perioperative period.

Proper nutrition is extremely important in IBD. A proper diet improves the effects of pharmacological treatment, reduces the number of complications, and prolongs periods of remission [34-36]. However, there is no diet that can be called "an intestinal diet". Nutritional recommendations depend on the location, extent, and activity of the disease, the patients' age, lifestyle, and nutrition, as well as the type of treatment and the patients' tolerance to food products. An appropriate model of nutrition should be individually prepared for each patient. In periods of remission there are no specific indications for the use of restrictive elimination diets, with the exception of food that causes problems in individual cases. Patients should be advised to have a normal, balanced diet and live a healthy lifestyle. In cases of aggravation of diarrhoea, low-fat and low-fibre diets are recommended. In patients with symptoms of 
intestinal stenosis, it is recommended that high-fibre products are limited.

Exacerbation of the disease may cause loss of appetite and nausea, and chronic diarrhoea can lead to dehydration and loss of electrolytes and vitamins. The nurse's duty is to identify the cause of reluctance to consume foods. This may be the disease itself and the fear of the aggravation of diarrhoea but it could also be a depressive reaction, anxiety, and fatigue due to the chronic disease. This state may be alleviated by encouraging patients to eat meals more frequently, in smaller amounts, and serving additional meals considering patients' food preferences. Nurses may suggest a consultation with a dietician concerning the composition and diversification of diet, considering the nutritional deficiencies occurring in a given patient. Nurses should also advise malnourished patients and patients in periods of exacerbation to supplement their diets with high-energy, balanced nutritional products available on the market.

Malnutrition is an important problem in IBD patients. It must always be considered in terms of qualitative and quantitative aspects. The most important mechanisms for the development of malnutrition are the following: malabsorption, loss of nutrients with stool, administered medicines, and the body's increased demand for energy due to chronic inflammation [37, 38]. Prevention of malnutrition and supplementation of deficiencies are the main goals of nutrition in growing children and adolescents. Nutritional deficiencies in these patients may lead to delayed sexual maturation, bone demineralisation, short stature, and consequently to psychosocial disorders [39].

It should be emphasised that malnutrition negatively affects the treatment process of patients and modulates the pharmacokinetics of drugs. Supplementing protein deficiency, which occurs in $25-80 \%$ of patients, is an important element of diet therapy [37, 38]. Anaemia and iron deficiency are the most important and the most common extraintestinal manifestations of IBD [34, $37,38]$. Anaemia reduces the quality of life of patients and requires intensive treatment, and this is why supplementation with vitamin $B_{12}$, folic acid, and iron is so important, depending on the cause of the deficiencies. Among the most commonly reported metabolic complications associated with bowel disease, osteopaenia and osteoporosis are mentioned [37]. The main cause of bone loss is energy malnutrition and malabsorption, especially malabsorption concerning fat-soluble vitamins, as well as long-term steroid therapy [37, 38]. Patients with severe and long-term course of the disease should use calcium and vitamin $\mathrm{D}_{3}$ supplements [37, 38]. During a period of exacerbation of symptoms, an appropriately balanced liquid diet is recommended. If there is no improvement or an intolerance of this form of nutrition occurs, enteral nutrition by oral route or by agavage entered via the nose into the stomach or small intestine is used. Due to lower risk of septic and metabolic complications, this type of nutrition, rather than total parenteral nutrition, is recommended. This type of feeding is natural, cheaper, well balanced, and safer for patients; moreover, it allows for a faster and easier return to a natural diet [40]. It may also be carried out at home, after consultation with a dietician and a doctor, involving community nurses. In Poland, enteral nutrition has been used in homes since January 2007. In accordance with the recommendations, different kinds of liquid formulations and artificial diets (synthetic, balanced diets) as well as supplements for different deficiencies, e.g. of proteins, vitamins, microelements, or lipids, are given. Preparations without fibre, lactose, and carrageenan covering the daily requirement for all components of the diet (energy, protein, fatty acids, carbohydrates, vitamins, and microelements) are recommended. Enteral feeding is recommended in the ECCO guidelines as an element supporting pharmacological treatment [3].

In emaciated patients with large deficits, in the case of intestinal stenosis, fistulas, and abscesses, after bowel resection surgery, and during the preparation for the surgery, it is preferable to use parenteral nutrition. In paediatric patients, parenteral nutrition is an alternative to drug therapy. It helps to gain a proper body weight, maintain the appropriate rate of growth, and is better tolerated than steroid therapy [41]. Parenteral nutrition is a direct intravenous administration of all necessary nutrients in an assimilable form. Usually, the so-called central line into a deep vein is used for this purpose. During parenteral nutrition, the digestive tract is excluded from its function, which allows for faster regression of inflammatory lesions and improvement in bowel function. However, exclusive long-term parenteral nutrition increases the risk of atrophy of the intestinal epithelium and the occurrence of septic complications. Therefore, especially in patients requiring long-term parenteral feeding, it is preferable to add natural feeding as much as possible. It is also possible to conduct and monitor parenteral nutrition at home, after proper training of patients, with active participation of nurses who are a part of the nutritional therapy team.

Probiotics are one of the elements combining pharmacotherapy and diet therapy. So far, there has been no conclusive evidence for the efficacy of probiotics in IBD, but many observations show their beneficial effects during therapy with antibiotics and during immunosuppressive therapy [42]. 


\section{Instrumental support Nursing procedures}

3.1. Nursing staff working with IBD patients should know the basic mechanisms of action and possible adverse events of drugs used. Patients with complications during the disease and in difficult life situations should have easier contact with specialised centres for the treatment of IBD patients.

The basic nursing procedures and the therapeutic process in IBD patients are not different from the standard diagnostic-therapeutic procedures in other chronic diseases. The advancement of knowledge, and the introduction of new technologies and drugs mean that continuous learning is one of the duties of nursing staff. The specificity of nursing care in IBD patients requires additional skills and experience, especially in the case of patients in a serious condition or with complications during the disease. Directing patients to the appropriate centres is the role of the staff of units that do not have such experience. This type of procedure practised at the level of medical care should be more and more common, also in the field of nursing care. This will be possible, as mentioned above, after the introduction of new communication standards based on modern technologies of communication.

\section{Procedures in pain management}

3.2. Through constant contact with patients, the nursing staff are able to observe the occurrence of pain and to assess the degree of severity, and then to take appropriate actions to ensure the proper treatment and support for patients in pain. Causes of pain in IBD may be multifactorial and not always linked to the exacerbated disease. Nurses, through communication and collaboration with MDT, may help patients to manage this symptom.

Abdominal pain is sometimes the first symptom of IBD, or it indicates exacerbation of the disease. It is a symptom that significantly affects the quality of life of patients. Determining its nature and managing it effectively is often a long process $[2,5,7,8]$.

Pain may be caused by severe inflammation, especially in the forms of the disease with intestinal stenosis, fistulas, and perianal fissures, or it may be a result of non-inflammatory mechanisms, such as post-surgical adhesions or concomitant functional disorders of the gastrointestinal tract $[4,7,43]$.

Extraintestinal factors including gallstones, renal calculi, pancreatitis, or joint and skin complications may also cause pain $[4,7,43]$.
Complaints of pain should trigger further investigations to uncover the source. This process includes a thorough collection of history and conducting a physical examination. Diagnostic imaging and endoscopy, as well as additional analyses, including some selected biochemical and haematological tests, may be also helpful.

The nursing staff spend the most time with patients in pain; therefore, their role in identifying, diagnosing the causes, and monitoring the efficacy of treatment is crucial. These tasks should be carried out jointly with the MDT, sometimes also with the help of a psychologist.

In order to assess pain properly, some scales for evaluating the degree of its severity may be used. For this purpose, the following scales are used most frequently: visual-analogue scale VAS, verbal rating scale VRS, and numerical rating scale NRS [44].

Nurses administrating drugs must have a wide knowledge of pharmacological pain control methods, adverse events, and drug interactions. The use of opioids for pain control is associated with numerous adverse events and a risk of addiction. Educating patients on the proper use of recommended drugs or other therapeutic methods is also the role of nurses. Determination of an algorithm of pain treatment in IBD could be useful in decision-making in clinical practice.

\section{Advanced nursing}

\section{Skills, competence, and permissions}

4.1. Nursing staff working in centres dealing with IBD patients should have extensive knowledge and experience in this field. They should also have clearly defined remits and permissions depending on their qualifications.

Nurses providing advanced care for IBD patients should have a bachelor's degree in nursing. They should also have completed additional courses: a qualifying course in conservative nursing, expert courses i.e., among others, a course in cardiopulmonary resuscitation, and several years of experience working in hospitals.

As part of their self-improvement and training, nursing staff should keep track of publications and the latest research reports in the field of nursing care.

The facility should organise internal training sessions on the principles of patient care, methods of treatment, and diagnostics. To an increased extent, Polish nurses should be able to participate in external training sessions, courses, conferences, and other forms of education.

Emotional and information support in the field of advanced nursing involves the same areas that have been discussed for fundamental nursing care. Experi- 
enced nurses should be able to advise both patients and staff from other units, engage in educational activities, especially within the support groups, and work closely with doctors specialising in the care of IBD patients.

It is advisable that the nurses who are leaders in the treatment centres for IBD patients have a master's degree. These people should coordinate the process of patient care in cooperation with the entire MDT. The exchange of experience between leaders of various centres and the development of common procedures may significantly improve patient care through the popularisation of knowledge and by establishing standards of conduct in the process of nursing care. These initiatives, such as the publication of the first Polish nursing guidelines for IBD patients, also serve the popularisation of knowledge concerning the importance of multidisciplinary care in optimising the diagnostic-therapeutic process. The introduction of modern methods of communication gives an opportunity for better monitoring of the therapy, and a rapid reaction in situations that are difficult for patients. However, this is not an easy task, since it requires intellectual, institutional, and financial support. The adoption of patronage over the process of development of the first Polish guidelines by the then National Consultant of Gastroenterology shows the importance of the issues discussed. Establishing organisational and scientific cooperation with leading centres of this type around the world is the next task that nursing staff from Polish treatment centres for IBD patients are facing. This has become possible through the inclusion of a group of Polish nurses into the structures of N-ECCO. Additionally, some efforts to initiate multi-centre studies evaluating different aspects of quality of life in the population of Polish patients have been started.

Creating a specialisation of nursing care for IBD patients in Poland, as is the case in leading countries, will be one of the objectives of the Working Group in the future.

\section{Instrumental support}

\section{Biological treatment - qualifications}

5.1. The nursing staff taking an active part in the therapeutic process, including biological treatment, should have the appropriate qualifications and skills to provide proper supervision and monitoring of patients, and to respond to possible adverse events of such treatment effectively and as fast as possible.

The progress in the pharmacotherapy of patients with inflammatory bowel disease that has been observed over the last dozen or so years results from the introduction of a new group of biologics into clinical practice. Proper conduct of the therapeutic process requires knowledge of the mechanisms of action of a drug, the principles of administration, the possible adverse events, as well as the standards for patient monitoring. Very often, clinical trials of new drugs that are not registered for routine pharmacological treatment are conducted in specialist centres. This requires additional qualifications and certifications, as well as careful observations and thorough keeping of medical records.

\section{Biological treatment - drug effects}

\subsection{Nurses caring for IBD patients know the mechanism of action of biologics as well as the possible adverse events (early and late).}

Adalimumab and infliximab are the biologics used currently in Poland for the treatment of IBD patients. They are administered mainly within the drug programmes funded by the National Health Fund. In the near future, in connection with the termination of patent protection, the so-called "biosimilar preparations", produced by manufacturers other than the manufacturers of the original formulations, will be introduced into clinical practice. The biologics registered in Poland are recombinant proteins with a low molecular weight and with monoclonal antibody characteristics. They show anti-inflammatory effects by blocking a mediator of inflammation, i.e. cytokine TNF- $\alpha$. They are effective both in remission induction and maintenance therapy $[3,6,8,9]$.

Infliximab is administered by intravenous infusion; therefore, there is a greater risk of early adverse events than in the case of subcutaneous administration of adalimumab. The most common adverse events include dyspnoea, hives, and headaches, which require a decision on the discontinuation of the infusion, reducing its flow, or the administration of additional drugs. During the intravenous infusion, some patients may experience symptoms of anaphylactic shock (severe allergic reaction), as well as delayed-type hypersensitivity (allergic reaction with subsequent infusions of the drug). These are states of immediate danger to life; therefore, it is necessary to follow the instructions for the drug administration and monitoring of patients' condition carefully. In such situations, one should immediately discontinue the infusion, notify the medical staff, and start conducting the necessary therapeutic procedures. Sometimes, patients treated with infliximab may have symptoms of serum sickness-like disease (including skin rash, joint pain, or fever), which usually leads to a decision of changing the treatment. The administration of adalimumab rarely leads to the occurrence of early adverse events. Such events mostly concern local skin reactions. 
The most serious late adverse effects associated with biological treatment result from an alternated immune response and manifest in the occurrence of viral, bacterial, and fungal infections that are often severe in their clinical course. Tuberculosis and opportunistic infections that are typical for patients with reduced immune response are the most threatening complications $[4,7-9,23]$. It should be remembered that the majority of patients before and during treatment with biologics also take other medications that weaken the immune response, such as glucocorticoids or immunosuppressive drugs.

\section{Biological treatment - preparation and administration of drugs}

5.3. The nursing staff caring for IBD patients know the principles for the preparation of a drug for administration in accordance with the Summary of Product Characteristics and additional instructions for a particular patient. They also monitor the administration of the drug and the patients' condition carefully and take appropriate actions in the event of adverse events.

Nurses giving the biological drugs must know that infliximab should be administered by an intravenous infusion lasting at least 2 hours, and the time to monitor the health status of patients should include an additional period of 1-2 hours after the end of the infusion, when severe reactions associated with the administration of the drug may occur. Emergency equipment must be located near patients. In some patients, especially in those with a history of allergic reactions related to the infusion, an antihistamine drug, hydrocortisone, and paracetamol are given before the actual infusion. It is advisable in such cases to reduce the infusion rate in order to minimise the risk of adverse events.

Adalimumab is a drug given subcutaneously. The time of drug administration is short, from $2 \mathrm{~s}$ to $5 \mathrm{~s}$. After the injection, one should press the injection site for $10 \mathrm{~s}$ using a swab, without rubbing or massaging. There may be a little bleeding that resolves spontaneously. Administration of adalimumab is simple and safe. After proper training on the rules of storage, patients may self-administer the drug at home, or even while travelling.

\section{Biological treatment - the role of coordinating nurses}

5.4. Nurses coordinating patient care at the site of biological treatment are responsible for ensuring that patients receive the drug in accordance with the protocol. Their task is also to ensure timely delivery of drugs from a chemist, proper storage control, and monitoring of the schedule of visits and indicated examinations in patients.

Proper organisation of work in a centre for biological treatment requires an efficient and economically justified ordering of drugs. This is associated with the necessity of continuous monitoring and modification of the biological treatment process, attention to fulfilling orders correctly, and storing the drugs in the centre properly. The practice shows that some patients, especially during a period of remission, do not follow the treatment recommendations and forget, or deliberately reduce, the administration of drugs. The same happens in the case of patients treated biologically within the drug programmes of the National Health Fund. Therefore, monitoring of the schedule of visits associated with the administration of drugs and the necessity to perform additional tests falls to coordinating nurses. Particular attention should be paid to proper treatment of outpatients. This requires systematic control and checking whether patients received the recommended dose of the drug in a timely manner [45].

\section{Fertility, pregnancy, and breastfeeding in inflammatory bowel disease}

5.5. In collaboration with other professionals, nurses should provide prenatal and perinatal care, as well as care during breastfeeding for patients and their partners.

Inflammatory bowel disease mostly affects young people in their reproductive years, so they have justified concerns about the chances of having healthy offspring. Research shows that the majority of women with IBD reported safe pregnancies, and gave birth to healthy babies [4].

Statistically people with IBD have fewer children than healthy couples. Temporary infertility may occur in men treated with salazopyrin. This effect is completely reversible after discontinuation of the drug or after changing to another drug from the group of 5-aminosalicylic acid derivatives [4]. Reduced fertility may result from the activity of the disease and its complications, such as perianal and entero-cutaneous fistulas, abscesses, and anal fissures. These are the complications that adversely affect sex life by lowering the comfort and the sense of sexual attractiveness. The related problems require understanding and tolerance from partners and sometimes also help from a psychologist or sexologist.

Adverse effects on fertility in both sexes may be due to surgery [7]. Active disease in some patients leads to 
impaired absorption and disturbed nutritional status, which in women may lead to cessation of ovulation as well as lower efficacy of oral contraceptives [46]. This is another important issue that should be discussed with patients. Planning conception at the remission of disease and using an optimal therapy throughout the pregnancy is the most important factor determining the proper course of pregnancy and delivery of a healthy baby [46]. Therapy to maintain remission should be continued because its exacerbation poses a greater risk to the foetus than an effective, sometimes even aggressive, therapy. Sometimes, patients, without consulting a gastroenterologist, through fear of the impact of drugs on the foetus, decide to discontinue treatment. Help in solving all the problems relating to the physical and psychological aspects of sexuality, effective contraception, pregnancy planning, and monitoring of the proper treatment during pregnancy is a very important aspect of holistic patient care provided in part by the nursing staff. This requires not only extensive knowledge but also proper organisation of patient care involving gastroenterologists, gynaecologists and obstetricians, sexologists, psychologists, and nursing staff in each of these fields. Identification of the existing problems and educational needs of patients by the nursing staff and their referral, if necessary, to appropriate specialists is the basis for the functioning of such MDTs. Close contact between the nursing staff and patients means that these issues are included in discussions, through which patients may gain all the necessary information. Providing appropriate educational materials in the form of brochures, handbooks, or reviews prepared by approved social organisations acting on behalf of IBD patients may also be helpful.

The nutritional status of pregnant IBD patients should be carefully monitored, and their diet should be properly balanced. Currently, it is believed that nutritional deficiencies of mothers are a factor in the risk of premature births and low birth weight of children. Following the diagnosis of malnutrition, a dietician should be involved in the therapeutic process. They will help in the selection of a well-balanced diet with a focus on the supplementation with calcium, vitamin D, iron, and folic acid as well as on the individual nutritional needs of patients.

In consultation with a gastroenterologist, a gynaecologist should determine the date and method of childbirth, which, depending on the current course of the disease and pregnancy, can be done vaginally or by caesarean section [7].

Patients should be informed that the use of certain drugs does not preclude breastfeeding $[4,46]$, and specific recommendations should be determined by the attending gastroenterologist.

\section{Fistulating inflammatory bowel disease}

5.6. Providing physical and mental comfort to patients is the duty of the nursing staff in fistulating IBD. This applies in particular to monitoring the hygienic recommendations, dressing the wound, and applying appropriate dressings, as well as psychological and educational support in each individual case.

Fistulating IBD mainly affects patients with CD. Fistulas are a pathological connection between the intestine and the skin (external fistulas) or other organs, most commonly the urinary bladder and/or the ureter, the vagina, or the adjacent intestinal loops (internal fistulas) [4]. Leakage of intestinal contents into the vagina, the urinary tract, or via the skin constitutes one of the symptoms. This is accompanied by local inflammation associated with pain and itching of the external orifice of the fistula, and often also by a systemic infection, e.g. recurrent cystitis or pyelonephritis. During the initial formation of a fistula, an abscess may be formed. It usually requires combination drug therapy and surgical intervention. This type of course is most common in patients with perianal location of the disease complicated by formation of perianal fistulas. They are formed from the rectal vaults or the penetration of the inflammatory process from ulceration involving the full thickness of the intestinal wall. The treatment of fistulas is a long process, requiring multidisciplinary care with the use of optimal pharmacological and surgical therapy and nursing care, including teams specialising in the treatment of chronic wounds. Fighting infection in the wound induced by a fistula requires debridement, application of antiseptics and dressings, as well as local and systemic antibiotic therapy, where appropriate. The principles of treatment of chronic wounds applicable for patients with fistulating IBD have recently been published in the form of the guidelines of the Polish Wound Healing Society [47]. Knowing these issues is essential in the work of the nursing staff specialising in the care of IBD patients.

In female patients with entero-vaginal fistulas, special attention should be paid to the care of the perineum to prevent skin maceration, chafes, or ulceration. For gentle and effective cleansing, professional preparations with neutral $\mathrm{pH}$ are recommended. It is recommended that the skin bedried carefully and lubricated with oil. Due to the unpleasant odour accompanying the fistula, patients may be offered help in the selection of appropriate urological pads or nappies. Patients with fistulating form of the disease may require hospitalisation in a surgical ward. The anxiety and fear caused in patients by this form of disease, the uncertain prog- 
nosis, and the prospect of hospitalisation require special commitment from the nursing staff in providing comprehensive support. Patients staying in the surgery ward feel extreme psychological discomfort due to the worsening of symptoms and the expected surgery. They also lose their sense of freedom, which is very often a result of disruption of intimacy caused by the disease.

The nursing staff who are experienced in caring for IBD patients may effectively make full contact with patients, give them a sense of safety, facilitate adaptation to the conditions of the ward, and answer questions and clarify doubts regarding the care and nursing. Under such circumstances, it is very important for patients to be provided with emotional support.

\section{Stoma}

\subsection{Patients with emerged stoma are a special group} of patients. All of them, regardless of age, gender, educational level, and occupation, require special attention and specialist care of MDTs.

Surgical treatment in UC is usually an ultimate therapeutic method, involving the removal of the large intestine in patients not responding to medical treatment and with severe life-threatening complications of the disease, such as massive haemorrhage or toxic megacolon. In the case of $\mathrm{CD}$, sparing surgeries are planned if necessary, since the removal of an affected part of bowel does not mean that the disease is cured $[3,4]$. In some operated patients, it is necessary to create a permanent or temporary stoma, which is a connection between the intestinal lumen and the surface of the body. This type of surgery is a traumatic experience for patients, and giving consent for stoma is sometimes a very difficult decision. It is also a challenge for professional, specialised nursing care.

Tasks concerning comprehensive care for patients during preparation for surgery, the perioperative period, and during the further course of the disease should be carried out by multidisciplinary teams including surgeons and gastroenterologists, nurses caring for IBD patients, stoma clinic nurses, psychologists, and dieticians. The "stoma" nurses in specialist centres are experts in caring for patients with entero-cutaneous fistulas. During the preoperative period, stoma nurses present patients with the surgical procedure and issues related to lifestyle after the surgery, demonstrate various types of equipment, and, together with a surgeon, determine the optimal location for the stoma on the abdomen. During the postoperative period, they help patients in mastering the principles of proper care of the stoma and the skin around the stoma, and discuss basic dietary recom- mendations. In long-term care, one should also consider social and psychological readaptation, and providing patients with a chance to return to normal family, social, and professional life. Participation in educational and informational meetings organised by the association acting on behalf of stoma patients - the Polish Stoma Association Pol-ilko - may be a great help for patients. Nursing staff specialising in the care of IBD patients should facilitate contact between a Stoma Clinic and a person with a stoma and encourage them to read the informational and educational offer of a support group.

\section{Faecal incontinence}

5.8. Nurses should collect relevant information, note the problem, and assess the impact of faecal incontinence on the quality of life of patients. Care must be provided individually, and in justified cases patients should be advised to consult a specialist. Nursing tasks include education and nursing as well as specialist interventions that improve the functioning of patients. During hospitalisation, it is important to ensure permanent access to toilets and respect the principles of privacy.

Inflammatory changes of the distal large intestine, perianal complications, and surgeries in this area can lead to faecal incontinence. Faecal incontinence has a significant impact on quality of life of patients, it leads to the limitation of activities of daily living and exclusion from social life, and generates a sense of shame and fear. During conversations with patients, the nursing staff should obtain detailed information on the symptoms to be able to help patients in obtaining optimal care and coping with the problems of physical and psychosocial nature. Care of perianal skin vulnerable to chafes is also important in the nursing care. Patients should be offered a selection of appropriate personal hygienic agents - absorbent materials in the form of pads, adult nappies, or liners that protect the skin but also eliminate odours that are unpleasant for patients and other people in their environment. The tasks of nurses should include, among others, supervision of the regular intake of prescribed (e.g. anti-diarrhoeal) drugs and adherence to a proper diet. In the treatment of faecal or urinary incontinence syndromes, rehabilitation plays an important role. Systematic training of appropriate muscle groups, conducted under the supervision of a physical therapist, may in some cases reduce the symptoms. After diagnosing the problem by the nursing staff, patients treated in specialist centres should be able to use this type of treatment as well as the help of a psychotherapist. 


\section{The prospects for the future of inflammatory bowel disease nursing}

The guidelines for nursing in IBD patients developed by the Working Group indicate the need for continuous improvement of knowledge and the exchange of experience by the nursing staff working in multidisciplinary teams involved in the care for IBD patients. Patients are usually young people suffering from a chronic, incurable disease with the risk of complications. Advances in research on the pathogenesis and multidisciplinary treatment of IBD also require continuous education of the nursing staff, while the introduction of new drugs and diagnostic procedures requires competence and good work organisation. Due to constant contact with patients in hospitals and in ambulatory conditions, the nursing staff also have a chance to monitor the disease and collect information that is necessary in the therapeutic process. When executing the mission of their profession, the nursing staff should provide patients with emotional, informational, and instrumental support. Working with IBD patients requires constant updating of knowledge and inpatient and outpatient treatment experience within the field of internal and surgical nursing, endoscopy, and treatment of chronic wounds. It also requires the ability to establish contact with patients and other employees of multidisciplinary therapeutic-caring teams. The specificity of working with IBD patients and implementation of programmes of biological treatment should be based on standards and procedures that are uniform across the country and consistent with the guidelines of appropriate European associations (ECCO, N-ECCO) and Polish guidelines for management of patients with $C D$ and $U C$ $[8,9]$. The Working Group believes that it is reasonable, taking other European countries as a model, to make efforts to create a new specialisation in nursing care for IBD patients. Promoting developed guidelines within the medical community and continuing the organisational and scientific cooperation in the framework of N-ECCO are other important objectives of the Working Group. Possibly, the planned multi-centre studies will allow us to assess the real impact of nursing care on the process of treatment of IBD patients in Poland.

\section{Acknowledgments}

The Working Group developing the guidelines would like to acknowledge the support as well as the organisational and substantive help of the contemporary National Consultant for Gastroenterology, Professor Grażyna Rydzewska.

The meeting of the Working Group, which was followed by the development of the guidelines, was possible thanks to the support of Abbvie.

\section{References}

1. O'Connor M, Bager P, Duncan J, et al. N-ECCO Consensus statements on the European nursing roles in caring for patients with Crohn's disease or ulcerative colitis. J Crohns Colitis 2013; 7: 744-64.

2. Van Assche G, Dignass A, Panes J, et al. The second European evidence-based Consensus on the diagnosis and management of Crohn's disease: definitions and diagnosis. J Crohns Colitis 2010; 4: 7-27.

3. Dignass A, Van Assche G, Lindsay JO, et al. The second European evidence-based Consensus on the diagnosis and management of Crohn's disease: current management. J Crohns Colitis 2010; 4: 28-62.

4. Van Assche G, Dignass A, Reinisch W, et al. The second European evidence-based Consensus on the diagnosis and management of Crohn's disease: Special situations. J Crohns Colitis 2010; 4: 63-101.

5. Dignass A, Eliakim R, Magro F, et al. Second European evidence-based consensus on the diagnosis and management of ulcerative colitis. Part 1: definitions and diagnosis. J Crohns Colitis 2012; 6: 965-90.

6. Dignass A, Lindsay JO, Sturm A, et al. Second European evidence-based consensus on the diagnosis and management of ulcerative colitis. Part 2: current management. J Crohns Colitis 2012; 6: 991-1030.

7. Van Assche G, Dignass A, Bokemeyer B, et al. Second European evidence-based consensus on the diagnosis and management of ulcerative colitis: special situations. J Crohns Colitis 2013; 7: 1-33.

8. Łodyga P, Eder P, Bartnik W, et al. Guidelines for the management of Crohn's disease. Recommendations of the Working Group of the Polish National Consultant in Gastroenterology and the Polish Society of Gastroenterology [Polish]. Prz Gastroenterol 2012; 7: 317-38.

9. Eder P, Łodyga M, Łykowska-Szuber L, et al. Guidelines for the management of ulcerative colitis. Recommendations of the Working Group of the Polish National Consultant in Gastroenterology and the Polish Society of Gastroenterology [Polish]. Prz Gastroenterol 2013; 8: 1-20.

10. Andrzejewska J, Talarska D. The quality of life in inflammatory bowel disease. The analysis and validation of a new research tool [Polish]. Prz Gastroenterol 2009; 4: 88-92.

11. Vidal A, Gomez-Gil E, Sans M, et al. Health-related quality of life in inflammatory bowel disease patients: the role of psychopathology and personality. Inflamm Bowel Dis 2008; 14: 977-83.

12. Talarska D, Strugała M. Nursing care of people with gastrointestinal diseases. In: Nursing in Internal Medicine [Polish]. Talarska D, Zozulińska-Ziółkiewicz D (red.). PZWL, Warszawa 2009; 147-75.

13. Andrews JM, Mountifield RE, Van Langenberg DR, et al. Unpromoted issues in inflammatory bowel disease: opportunities to optimize care. Intern Med J 2010; 40: 173-82.

14. Clarke L. So what exactly is a nurse? J Psychiatr Mental HLT 2006; 13: 388-94.

15. Ślusarska B, Zarzycka D, Dobrowolska B. Perception of professional identity by nurses [Polish]. Problemy Pielęgniarstwa 2007; 15: 147-56. 
16. Ślusarska B, Dobrowolska B, Zarzycka D. The theoretical basis of the category of "care" in nursing [Polish]. Problemy Pielęgniarstwa 2008; 16: 384-9.

17. Dobrowolska B. Nursing and holistic nursing care [Polish]. Pielęgniarstwo XXI Wieku 2002; 1: 14-9.

18. Ardizzone S, Puttini PS, Cassinotti A, et al. Extraintestinal manifestations of inflammatory bowel disease. Dig Liver Dis 2008; 40 (Suppl 2): S253-9.

19. Costa F, Mumolo MG, Bellini M, et al. Role of faecal calprotectinas non-invasive marker of intestinal inflammation. Dig Liver Dis 2003; 35: 642-7.

20. Hernandez-Sampelayo P, Seoane M, Oltra L, et al. Contribution of nurses to the quality of care in management of inflammatory bowel disease: a synthesis of the evidence. J Crohns Colitis 2010; 4: 611-22.

21. Ginsberg GG, Kochman ML, Norton I, et al. Clinical gastrointestinal endoscopy [Polish]. Vol. 1. Medipage, Warszawa 2009; 105-10.

22. Sęk H, Cieślak R. Social support, stress and health [Polish]. PWN, Warszawa 2005.

23. Rahier JF, Ben-Horin S, Chowers Y, et al. European evidence based consensus on the prevention, diagnosis and management of opportunistic infections in inflammatory bowel disease. J Crohns Colitis 2009; 3: 47-91.

24. Krajewska-Kułak E, Panek W. Interpersonal communication in nursing [Polish]. Czelej, Lublin 2003.

25. Husain A, Triadafilopoulos G. Communicating with patients with inflammatory bowel disease. Inflamm Bowel Dis 2004; 10: 444-50.

26. Nightingale AJ, Middleton W, Middleton SJ, Hunter JO. Evaluation of the effectiveness of a specialist nurse in the management of inflammatory bowel disease (IBD). Eur J Gastroenterol Hepatol 2000; 12: 967-73.

27. McCabe C. Nurse-patient communication: an exploration of patients' experiences. J Clin Nurs 2004; 13: 41-9.

28. Smith GD, Watson R, Roger D, et al. Impact of a nurse-led counselling service on quality of life in patients with inflammatory bowel disease. J Adv Nurs 2002; 38: 152-60.

29. Miller L, Caton S, Lynch D. Telephone clinic improves quality of follow-up care for chronic bowel disease. Nurs Times 2002; 98: 36-8.

30. Casellas F, Lopez-Vivancos J, Badia X, et al. Influence of inflammatory bowel disease on different dimensions of quality of life. Eur J Gastroenterol Hepatol 2001; 13: 567-72.

31. Philpott JR. Transitional care in inflammatory bowel disease. Gastroenterol Hepatol 2011; 7: 26-32.

32. Baldassano R, Ferry G, Griffiths A, et al. Transition of the patient with inflammatory bowel disease from pediatric to adult care: recommendations of the North American Society for Pediatric Gastroenterology, Hepatology and Nutrition. J Pediatr Gastroenterol Nutr 2002; 34: 245-8.

33. Politi P, Bodini P, Mortilla MG, et al. Communication of information to patients with inflammatory bowel disease: a European Collaborative Study in a multinational prospective inception cohort. J Crohns Colitis 2008; 2: 226-32.

34. Baczewska-Mazurkiewicz D, Rydzewska G. Nutritional problems in inflammatory bowel disease patients [Polish]. Prz Gastroenterol 2011; 6: 69-77.
35. Rajendran N, Kumar D. Role of diet in the management of inflammatory bowel disease. World J Gastroenterol 2010; 16 : 1442-8.

36. Lucendo AJ, De Rezende LC. Importance of nutrition in inflammatory bowel disease. World J Gastroenterol 2009; 15: 2081-8.

37. Grzymisławski M. Metabolic complications of LeśniowskiCrohn's disease [Polish]. Prz Gastroenterol 2006; 1: 61-4.

38. Eiden KA. Nutritional considerations in inflammatory bowel disease. Pract Gastroenterol 2003; 5: 33-54.

39. Roberts J. Understanding inflammatory bowel disease in children. Nurs Times 2007; 103: 28.

40. Rydzewska G. Selected aspects of enteral and parenteral nutrition in adults [Polish]. Ped Współ Gastroenterol Hepatol Żyw Dz 2004; 6: 461-5.

41. Wilschanski M, Sherman P, Pencharz P, et al. Supplementary enteral nutrition maintains remission in paediatric Crohn's disease. Gut 1996; 38: 543-8.

42. Strus M, Drzewiecki A, Heczko P. Possible applications of probiotics in the treatment of chronic non-specific inflammatory bowel diseases [Polish]. Gastroenterol Pol 2005; 12: 225-8.

43. Bielefeldt K, Davis B, Binion DG. Pain and inflammatory bowel disease. Inflamm Bowel Dis 2009; 15: 778-88.

44. Ulatowska A, Bączyk G, Lewandowska H. Review of the literature on post-operative pain and the quality of nursing care [Polish]. Piel Chir Angiol 2012; 1: 7-12.

45. Duncan J, Caulfield S, Clark A, et al. A multidisciplinary virtual biologic clinic: is it worthwhile? J Crohns Colitis 2010; 4: S70.

46. Vermeire S, Carbonnel F, Coulie PG, et al. Management of inflammatory bowel disease in pregnancy. J Crohns Colitis 2012; 6: 811-23.

47. Jawień A, Bartoszewicz M, Przondo-Mordarska A, et al. Guidelines for local and systemic treatment of infected chronic wounds [Polish]. Leczenie Ran 2012; 9: 59-75.

Received: 15.08.2014

Accepted: 31.08 .2014 